\title{
Oral Presentations
}

\section{New perspectives on therapeutic immune tolerance.}

\begin{tabular}{l|l}
\hline OP0002 & IMMTOR NANOPARTICLES ENHANCE THE \\
TOLEROGENIC ENVIRONMENT OF THE LIVER IN MICE
\end{tabular}

P. Ilyinskii ${ }^{1}$, C. Roy' ${ }^{1}$ J. Leprevost ${ }^{1}$, K. Kishimoto ${ }^{1} .^{1}$ Selecta Biosciences, Biology, Watertown, United States of America

Background: Tolerogenic ImmTOR biodegradable nanoparticles encapsulating rapamycin have been shown to mitigate the formation of anti-drug antibodies against pegadricase, a pegylated uricase enzyme, which enabled monthly dosing and sustained reduction of serum uric acid levels in a Phase 2 clinical trial of SEL-212, a combination of pegadricase + ImmTOR, in patients with symptomatic gout with hyperuricemia. Prior mechanism of action studies showed selective biodistribution of ImmTOR to the spleen and liver following intravenous (IV) administration in mice. In the spleen, ImmTOR has been demonstrated to induce tolerogenic dendritic cells and antigen-specific regulatory $T$ cells and inhibit antigen-specific B cell activation. Splenectomized mice showed a partial but incomplete abrogation of the tolerogenic immune response mediated by ImmTOR.

Objectives: Here we evaluated the ability of ImmTOR to enhance the tolerogenic environment in the liver.

Results: All the major resident populations of liver cells, including liver sinusoidal endothelial cells (LSECs), Kupffer cells (KC), stellate cells (SC), and hepatocytes, actively took up fluorescent-labeled ImmTOR particles, which resulted in downregulation of $\mathrm{MHC}$ class II and co-stimulatory molecules and upregulation of the PD-L1 checkpoint molecule. The LSEC, known to play an important role in hepatic tolerance induction, emerged as a key target cell for ImmTOR. The tolerogenic environment led to a multi-pronged modulation of hepatic $\mathrm{T}$ cell populations, resulting in an increase in $\mathrm{T}$ cells with a regulatory phenotype, upregulation of PD-1 on $\mathrm{CD}^{+}$and $\mathrm{CD} 8^{+} \mathrm{T}$ cells, and the emergence of a large population of $\mathrm{CD} 4^{-} \mathrm{CD}^{-}$(double negative) $\mathrm{T}$ cell population. Modulation of $\mathrm{T}$ cell phenotype was seen to a lesser extent after administration by empty nanoparticles, but not free rapamycin. The upregulation of PD-1, but not the appearance of double negative T cells, was inhibited by antibodies against PD-L1 or CTLA-4

Conclusion: These results suggest that the liver may contribute to the tolerogenic properties of ImmTOR in mitigating anti-drug antibody responses to biologic therapies, such as pegadricase.

Disclosure of Interests: Petr llyinskii Shareholder of: Selecta Biosciences, Employee of: Selecta Biosciences, Christopher Roy Shareholder of: Selecta Biosciences, Employee of: Selecta Biosciences, Julie LePrevost Shareholder of: Selecta Biosciences, Employee of: Selecta Biosciences, Kei Kishimoto Shareholder of: Selecta Biosciences, Employee of: Selecta Biosciences DOI: 10.1136/annrheumdis-2021-eular.1920

\section{Autoimmune diseases by abnormal T cell function}

\begin{tabular}{|l}
\hline OP0003 \\
AUTOREACTIVE CD4+T CELLS AND THEIR TCR \\
REPERTOIRE IN PR3-ANCA ASSOCIATED VASCULITIS
\end{tabular}

R. Kumar ${ }^{1}$, N. Yoosuf ${ }^{1}$, A. Bartoletti ${ }^{2}$, A. Avik ${ }^{3}$, B. Raposo ${ }^{1}$, A. Jonasdottir ${ }^{4}$, B. Lövström ${ }^{1}$, K. Chemin $^{1}$, A. Bruchfeld ${ }^{4}$, I. Gunnarsson ${ }^{1}$, V. Malmström ${ }^{1}$. ${ }^{1}$ Karolinska Institutet, Division of Rheumatology, Department of Medicine, Stockholm, Sweden; ${ }^{2}$ The University of Pavia, Medicine, Pavia, Italy; ${ }^{3}$ Danderyds hospital, Rheumatology, Stockholm, Sweden; ${ }^{4}$ Karolinska University Hospital, CLINTEC, Stockholm, Sweden

Background: ANCA-associated vasculitis (AAV) with proteinase 3 (PR3) ANCA is genetically associated with HLA-DP [1], is often relapsing in nature, and has a predisposition for kidneys, lungs and ear-nose-throat involvement [2]. Despite the presence of PR3+ANCA, indicating CD4+T-cell help in the disease, the knowledge about autoreactive CD4+T cells is scarce. Activated $T$ cells have been shown at site of inflammation [3] and involvement of proinflammatory cytokines in circulation is also reported $[4,5]$.

Objectives: Identification of autoreactive T cells may help to identify the drivers of the immune responses and chronicity. We therefore aimed to investigate PR3-specific CD4+T-cell responses in peripheral blood of AAV patients with a focus on both phenotype and T-cell receptor (TCR) repertoires.

Methods: The study included sixty-six patients: 26 with active PR3 autoantibody+ AAV, 21 with inactive but PR3+ AAV and 19 with inactive PR3- AAV. In-vitro cultures with PR3 protein were established to assess antigen-specific cytokine responses in a 3-color fluorospot assay. Deep immunophenotyping was performed by flow cytometry. Antigen-responsive CD4+ T cells were isolated and single cell TCR $\alpha \beta$ sequences were generated and analyzed from PR3+ AAV patients $(n=5)$ using a previously published protocol [6].

Results: PBMCs from AAV patients demonstrated an HLA-DP associated cytokine responses to PR3 stimulation including IFN- $\gamma$ and IL-10, but not IL-17A This T-cell autoreactivity was found to be confined to a highly differentiated CD4+ T cell population characterized by perforin and GPR56 expression, implicating a cytotoxic feature of the response. Active disease involved a reduction in expression of several markers associated with cytotoxicity amongst the CD4+GPR56+ $T$ cells. Their frequency was also negatively associated with the doses of prednisolone. A similar phenotype was shared with $\mathrm{T}$ cells activated by human cytomegalovirus (HCMV) peptides in the same patient cohort. Single cell sequencing of paired alpha beta T-cell receptors (TCRs) revealed different patterns of gene usage between PR3 and HCMV reactive T cells. Moreover, we could identify shared (public) PR3-reactive T-cell clones between different HLA-DPB1*04:01+ patients.

Conclusion: PR3 is an autoantigen which provokes ANCA responses in AAV patients. Our study identified PR3-reactive CD4+ T cells at the level of their phenotype and TCR repertoire. The autoreactive CD4+ T cells, present in both active and inactive disease, implicate chronic antigen exposure and the persistence of long-lived T-cell clones. The presence of public autoreactive clones between HLA-DPB ${ }^{*} 04: 01$ + patients suggests an active role for these cells in pathogenesis of AAV and validates the link with predisposed genotype.

\section{REFERENCES:}

[1] Lyons PA, Rayner TF, Trivedi S, Holle JU, Watts RA, Jayne DR, et al. Genetically distinct subsets within ANCA-associated vasculitis. New England Journal of Medicine. 2012; 367(3):214-223.

[2] Kumar Sharma R, Lövström B, Gunnarsson I, Malmström V. Proteinase 3 autoreactivity in Anti-Neutrophil Cytoplasmic Antibody-associated vasculitis-immunological versus clinical features. Scandinavian Journal of Immunology. 2020:e12958.

[3] Wilde B, Thewissen M, Damoiseaux J, van Paassen P, Witzke O, Tervaert JWCJAr, et al. T cells in ANCA-associated vasculitis: what can we learn from lesional versus circulating T cells? 2010; 12(1):204.

[4] Hoffmann JC, Patschan D, Dihazi H, Müller C, Schwarze K, Henze E, et al. Cytokine profiling in anti neutrophil cytoplasmic antibody-associated vasculitis: a cross-sectional cohort study. Rheumatology international. 2019; 39(11):1907-1917.

[5] Berti A, Warner R, Johnson K, Cornec D, Schroeder D, Kabat B, et al. Circulating Cytokine Profiles and ANCA Specificity in Patients with ANCA-Associated Vasculitis. Arthritis \& rheumatology (Hoboken, NJ). 2018; 70(7):1114.

[6] Han A, Glanville J, Hansmann L, Davis MM. Linking T-cell receptor sequence to functional phenotype at the single-cell level. Nature biotechnology. 2014 32(7):684-692.

Disclosure of Interests: None declared

DOI: 10.1136/annrheumdis-2021-eular.41

\begin{tabular}{|l|l}
\hline OP0004 & AUTOIMMUNE AND INFLAMMATORY \\
MANIFESTATIONS IN COMMON VARIABLE \\
IMMUNODEFICIENCY DISORDERS
\end{tabular}

K. López-Aldabe ${ }^{1}$, L. Hidalgo ${ }^{2}$, A. Antoli ${ }^{2}$, G. Rocamora ${ }^{2}$, X. Corbella ${ }^{2}$, X. Solanich' ${ }^{1}{ }^{1}$ Bellvitge University Hospital, Bellvitge Biomedical Research Institute-IDIBELL, Internal Medicine, L'Hospitalet de Llobregat, Spain; ${ }^{2}$ Bellvitge University Hospital, Bellvitge Biomedical Research Institute-IDIBELL, Internal Medicine, Hospitalet de Llobregat, Spain

Background: Common variable immunodeficiency (CVID) disorders are the second most frequent immunodeficiency worldwide and autoimmune diseases (AD) are present in $20 \%$ of such patients, cytopenia being the most frequent manifestation [1]. Defects in central and peripheral tolerance, activation/proliferation of $\mathrm{B}$ cells, and hypogammaglobulinemia are key features of the disease, along with a reduction in CD4+T cells, abnormalities in Treg and defective secretion of regulatory cytokines, that could perpetuate autoimmune - autoinflammatory phenomena.

Objectives: To describe immune and inflammatory disorders in our CVID cohort. Methods: Retrospective analysis of 33 patients who fulfill the European Society for Immunodeficiencies (ESID) Registry - Work criteria for CVID diagnosis [2] treated in the immunodeficiency unit of our tertiary university hospital. After getting an informed consent form, medical records were revised to obtain clinical, analytical and immunological data.

Results: Of the 33 CVID patients analysed, 11 had some autoimmune/ inflammatory manifestation. Seven patients presented autoimmune thrombocytopenia (AIT), and one of them also had non-severe neutropenia. Two patients also had seronegative spondyloarthropathy, one patient had cutaneous psoriasis, and 